\title{
A SIMPLE FORM OF NITROMETER FOR THE ASSAY OF SPIRIT OF NITROUS ETHER.
}

THEODORE J. BRADLEY.

The nitrometers on the market have been designed for general use and not

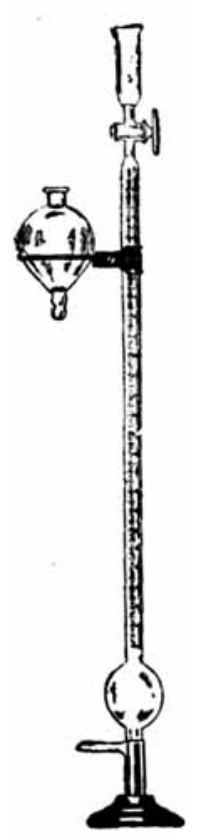
particularly for the assay of Spirit of Nitrous Ether, and they are, generally, expensive and complicated and more or less troublesome to use. During the past year the writer has had occasion to make a number of assays of this preparation and a special form of instrument was devised for this work.

The following features are found in this new form of nitrometer: it agrees in all essentials with the official general description, given on page 576 of the Pharmacopocia; it is simple and compact and of good appearance; it is complete, so that when one is ordered it is received ready for use; and its cost is moderate.

The instrument, as shown, consists of a glass tube about 16 inches long and graduated, from the top downward, to $50 \mathrm{cc}$. in fifths. At the top this graduated tube is contracted and has a stopcock connecting it with a cylindrical funnel which is also graduated at $5 \mathrm{cc}$. and $10 \mathrm{cc}$. The graduated tube, below the graduation, is expanded to form a bulb of about $75 \mathrm{cc}$. capacity, and below this there is a side tube with an open end to be connected with a leveling bulb. The bottom of the instrument is closed and it stands on a removable base, preferably of iron. The leveling bulb is connected with the side tube by about two feet of flexible rubber tubing and is supported by a clamp which is attached to the graduated tube and easily adjusted at any height.

This piece of apparatus has the same general appearance as Schiff's nitrometer and is a modification of that instrument. No great originality is claimed for it but it has proven to be well adapted for its special use. The instrument shown was made to order for $\$ 3.50$ by a firm of apparatus manufacturers in New York City. I have no doubt but that this price will be lowered when it is catalogued and made in quantities for regular stock.

\section{DISCUSSION.}

Dr. Bradleys-This ends the paper, but I should like to demonstrate the nitrometer and have the members discuss it. (Dr. Bradley here demonstrated the use of the instrument.)

Every one understands, I hope, that I have no financial interest in the sale of this instrument. This goes without saying, but I have said it just the same. Not many pharmacists realize that the assay of nitrous ether is a very simple operation, if we have a nitrometer. The actual work can be done inside of ten minutes, followed by a necessary wait of about a half an hour for the complete generation of the gas, and its change to room-temperature.

To fill this nitrometer or any form of a similar general character, we fill the leveling bulb about half full. There is an immediate passage of the liquid to the expansion bulb. It is necessary to stop at this point and expel air bubbles from the rubber tube. This can be done by squeezing it tightly, several times. (Indicating.) Then, if the leveling bulb is raised, the salt solution will fill the nitrometer. When the graduated tube is exactly filled we close the stopcock and lower the leveling bulb. 
The instrument is now ready for use. In the official process the first step is to shake the sample of spirit of nitrous ether with a small amount of potassium bicarbonate to neutralize any free acid. Then an ordinary $100 \mathrm{cc}$. measuring flask is weighed and about thirty grams of the sample are introduced and it is weighed again. Then we bring the volume of the liquid to $100 \mathrm{cc}$. with alcohol and mix. I have already done this part of the work and we can go right on with the assay. For ordinary store work you will find that the instrument is accurately graduated. We measure $10 \mathrm{cc}$. of the diluted sample in the funnel. This is then allowed to enter the graduated tube, being careful that no air follows. It may be safer to allow the last drop to remain in the funnel tube. It will be washed in by the first reagent. You thereby avoid the risk of air getting into it. $10 \mathrm{cc}$. of potassium iodid test solution are next measured in the funnel which is a large excess, and not a quantitative amount, and is allowed to enter the graduated tube. A slight reaction immediately ensues. And here again it is necessary to take care that no air gets in the nitrometer or we should have to start over again. The last reagent is $10 \mathrm{cc}$. of normal sulphuric acid introduced in the same manner as the preceding. I have always considered that it is unfortunate that normal sulphuric acid is specified as this is not found in many drug stores and it is troublesome to make. What is needed is but a weak sulphuric acid of about five per cent strength. The normal acid is of about this strength, but the same result is obtained more easily by using a mixture of equal parts of diluted sulphuric acid and water. The pharmacist who is not a well trained chemist might think that the normal strength of the acid is essential, but it is not. There is a large excess of the acid and as soon as it enters there is a violent reaction and our work is done.

It is necessary to allow the instrument to stand for from one-half to one hour. This is directed in the pharmacopoia to allow for the completion of the reaction, and for the gas to come to the room-temperature. Then it is necessary at the expiration of that time to bring the liquid in the bulb to the same level as the liquid within the graduated tube so that we obviate any difference of pressure between the gas inside of the tube and the air on the outside. Then we read the number of cubic centimeters of gas in the tube and calculate the percentage of ethyl nitrite from our first weighing, and this volume, by directions that are given in the pharmacopœia. It is necessary to observe the temperature, and if there is a considerable difference between the room-temperature, and $25^{\circ} \mathrm{C}$, a correction must be made, and in this correction the Pharmacopoia says, change the percentage calculated by "one-third of one per cent," of itself for each degree of difference in temperature, and to a person not accustomed to using these figures, that one-third of one per cent of a percentage is very confusing. We calculate a percentage and then we find one-third per cent of that percentage. If the pharmacopoia were changed to read, one three-hundredth of the percentage first calculated, which is exactly the same thing, it would be much clearer.

A very slight correction needs to be made, sometimes, for variations in pressure but this is not necessary for ordinary work. Before I stop, I will call your attention to this expansion bulb. If it were not there some of the sample and reagents would pass over into the rubber tube and perhaps some of the gas would escape through the leveling bulb.

Chairman Ruddiman: I would perhaps make one suggestion. In that apparatus, when you put in your salt solution, you have to be careful not to allow any air in there. Would it not help to have a graduation in this contracted part and then have the five and ten cc. measured from that graduation? In that way you would not run quite so much of a risk.

Mr. BradLey: I thought of that very thing, but left it out, as it was an additional expense, and because in making my own determinations, I measure the sample with a pipette and that drop is of no consequence. For ordinary work in manufacturing for which the pharmacist would use this instrument, that drop is so small, that it would not materially affect the result, and it can be reduced to a negligible quantity with a little practise.

A Memвer: Just one little detail I would like to ask about, and that is whether or not it is necessary to shake the nitrometer after the solutions have been added; ordinarily is it considered necessary?

Mr. Bradley: The pharmacopoeia specifies that detail, and I have compared results obtained with and without shaking and find them to be the same. 
We introduce the lightest liquid first and the heavier reagents on being added start to sink through it, but they mix on their way down and the shaking is unnecessary. It is very difficult to shake any nitrometer when making an assay and I have stopped doing it.

\section{THE ANALYSIS OF EMULSIONS.*}

CHARLES H. LAWALL AND LEROY FORMAN.

The analysis of emulsions by the drug analyst is a branch of work not generally welcomed, because of the lack of specific information on the subject, in books devoted to the analysis of pharmaceutical preparations. Even such recent books as "The Analysis of Drugs and Medicines" by Nelson, and "The Qualitative Analysis of Medicinal Preparations" by Fuller, contain little or no specific information on the subject.

The necessity for doing some practical work along this line, recently led us to experiment with the method known as the Gottlieb Roese Method, which has been used successfully for some time in the estimation of fat in dairy products, and the results were so surprisingly successful, when working upon known mixtures, that the method is suggested for routine work in this connection.

As officially described in the Proceedings of the A. O. A. C. for 1909, Bulletin 132 of the Bureau of Chemistry, U. S. Dept. of Agriculture, an extraction apparatius known as a Rohrig tube, which is now a standard piece of apparatus, is directed to be used. We have found, however, that excellent results can be obtained by the use of a glass graduated cylinder, and the evaporation of an aliquot part of the extraction liquid, although the method as described in full may be used if desired.

The modified method used by us is as follows:

Prepare a mixture of the emulsion in distilled water, so that each $100 \mathrm{cc}$. of the liquid contains $40 \mathrm{gm}$. of the emulsion. Take two $100 \mathrm{cc}$. graduated cylinders and in one, place $10 \mathrm{cc}$. of the diluted emulsion and in the other, place $5 \mathrm{cc}$. of the diluted emulsion and $5 \mathrm{cc}$. of water. To each cylinder then add the following reagents in the order named, agitating thoroughly after each addition :

$1 \mathrm{cc}$. stronger ammonia water.

10 cc. alcohol U. S. P.

25 cc. ether U. S. P.

25 cc. petroleum benzin U. S. P.

After the addition of the petroleum benzin, the agitation should be continuous for 10 minutes, after which the cylinders should be allowed to stand until the liquids have separated into two layers with a sharp dividing line, (this requires from 15 minutes to 1 hour). Then having observed the exact volume of the upper layer, draw off exactly one-half and transfer to a flat-bottomed glass capsule and evaporate quickly on a water bath to constant weight. In one of the duplicates the resulting fat will be from $2 \mathrm{gm}$. of the emulsion, in the other from $1 \mathrm{gm}$., which gives a satisfactory check upon the thoroughness of the extraction.

* Read before Scientific Section at Detroit Meeting. 